\title{
Vertical structure of the near-surface expanding ionosphere of comet 67P probed by Rosetta
}

\author{
K. L. Heritier, ${ }^{1 \star}$ P. Henrii, ${ }^{2}$ X. Vallières, ${ }^{2}$ M. Galand, ${ }^{1}$ E. Odelstad, ${ }^{3}$ A. I. Eriksson, ${ }^{3}$ \\ F. L. Johansson, ${ }^{3}$ K. Altwegg, ${ }^{4}$ E. Behar, ${ }^{5}$ A. Beth, ${ }^{1}$ T. W. Broiles, ${ }^{6}$ J. L. Burch, ${ }^{6}$ \\ C. M. Carr, ${ }^{1}$ E. Cupido, ${ }^{1}$ H. Nilsson, ${ }^{5}$ M. Rubin ${ }^{4}$ and E. Vigren ${ }^{3}$ \\ ${ }^{1}$ Department of Physics, Imperial College London, Prince Consort Road, London SW7 2AZ, UK \\ ${ }^{2}$ LPC2E, CNRS, Université d'Orléans, F-45100 Orléans, France \\ ${ }^{3}$ Swedish Institute of Space Physics, Angström Laboratory, Lägerhyddsvägen 1, SE-752 37 Uppsala, Sweden \\ ${ }^{4}$ Physikalisches Institut, University of Bern, Sidlerstrasse 5, CH-3012 Bern, Switzerland \\ ${ }^{5}$ Swedish Institute of Space Physics, PO Box 812, SE-981 28 Kiruna, Sweden \\ ${ }^{6}$ Southwest Research Institute, PO Drawer 28510, San Antonio, TX 78228-0510, USA
}

Accepted 2017 June 8. Received 2017 June 7; in original form 2017 March 31

\begin{abstract}
ABSTRA CT
The plasma environment has been measured for the first time near the surface of a comet. This unique data set has been acquired at 67P/Churyumov-Gerasimenko during ESA/Rosetta spacecraft's final descent on 2016 September 30. The heliocentric distance was 3.8 au and the comet was weakly outgassing. Electron density was continuously measured with Rosetta Plasma Consortium (RPC)-Mutual Impedance Probe (MIP) and RPC-LAngmuir Probe (LAP) during the descent from a cometocentric distance of $20 \mathrm{~km}$ down to the surface. Data set from both instruments have been cross-calibrated for redundancy and accuracy. To analyse this data set, we have developed a model driven by Rosetta Orbiter Spectrometer for Ion and Neutral Analysis-COmetary Pressure Sensor total neutral density. The two ionization sources considered are solar extreme ultraviolet radiation and energetic electrons. The latter are estimated from the RPC-Ion and Electron Sensor (IES) and corrected for the spacecraft potential probed by RPC-LAP. We have compared the results of the model to the electron densities measured by RPC-MIP and RPC-LAP at the location of the spacecraft. We find good agreement between observed and modelled electron densities. The energetic electrons have access to the surface of the nucleus and contribute as the main ionization source. As predicted, the measurements exhibit a peak in the ionospheric density close to the surface. The location and magnitude of the peak are estimated analytically. The measured ionospheric densities cannot be explained with a constant outflow velocity model. The use of a neutral model with an expanding outflow is critical to explain the plasma observations.
\end{abstract}

Key words: plasmas - methods: data analysis - Sun: UV radiation -comets: individual: 67P.

\section{INTRODUCTION}

After a 10-yr journey, followed by a 2-yr escort, the iconic ESA/Rosetta mission ended with a splendid final descent on comet 67P/Churyumov-Gerasimenko (Churyumov \& Gerasimenko 1972) on 2016 September 30 and returned its final data packet at 10:39 UT. It is the second man-made object to ever land on a comet after its lander Philae was deployed in 2014 November (Bibring et al. 2007, 2015). The past 2 yr have been ground breaking in terms of cometary science. While previous cometary missions were limited to flybys, the Rosetta mission was able to escort comet $67 \mathrm{P}$ and assess the

^E-mail: k.heritier15@imperial.ac.uk evolution of the cometary coma over a wide range of heliocentric distances (Glassmeier et al. 2007a). The Rosetta spacecraft encountered comet 67P in 2014 August at 3.6 au from the Sun. It escorted it from the arrival to perihelion in summer 2015 (1.24 au), and finally, to the end of mission in 2016 September at 3.8 au from the Sun. Such a high heliocentric distance implies low neutral outgassing. However, the measured neutral and ion densities were high due to the proximity of the spacecraft to the nucleus in its final hours.

The Rosetta Plasma Consortium (RPC) instruments monitored the cometary plasma during the full mission. Among them, the RPC-Mutual Impedance Probe (MIP) (Trotignon et al. 2007) and the RPC-LAngmuir Probe (LAP) (Eriksson et al. 2017) were able to derive ionospheric densities and electron temperatures. At large heliocentric distances, the electron density was found to be 
inversely proportional to the cometocentric distance, up to $250 \mathrm{~km}$ from the nucleus, and correlated to the neutral density diurnal variations (Edberg et al. 2015; Galand et al. 2016). The bulk of the electron population was associated with a temperature of 5-10 eV (Odelstad et al. 2015; Galand et al. 2016; Eriksson et al. 2017) at low cometocentric distances $(10-30 \mathrm{~km})$. This population is mixed with suprathermal electrons that have energies that can reach 100$200 \mathrm{eV}$ (Clark et al. 2015). These suprathermal electrons were probed by RPC-Ion and Electron Sensor (IES) (Burch et al. 2007). Solar wind fluxes or photoelectrons cannot explain this range of energy. Acceleration mechanisms, such as compression through the ambipolar electric field (Madanian et al. 2016) or heating through lower hybrid waves (Broiles et al. 2016), have to be invoked to explain the tail of the electron energy distribution. These suprathermal electrons contribute as ionizers for the neutral gas. Implementing them in ionospheric models makes possible to explain the total electron densities probed by RPC-MIP and RPC-LAP (Galand et al. 2016). Mixed with the electrons, the ion population at the location of Rosetta was largely composed of cometary ions (Nilsson et al. 2015a,b; Fuselier et al. 2016), as detected by the RPC-Ion Composition Analyzer (Nilsson et al. 2007) and the Rosetta Orbiter Spectrometer For Ion and Neutral Analysis (ROSINA)/Double Focusing Mass Spectrometer (Balsiger et al. 2007). At high heliocentric distances ( $>2.5 \mathrm{au}$ ), when the neutral outgassing is low, the solar wind is present and visible on RPC-ICA and RPC-IES spectra and has access to the surface of the nucleus (Wurz et al. 2015; Nilsson et al. 2017). The magnetic field of solar wind origin is monitored by the RPC-MAGnetometer (Glassmeier et al. 2007b), and enables the initial phase of the pick-up process of the cometary ions (Glassmeier 2017).

During the final descent, the RPC instruments measured cometary plasma until the touch down. This set of data is unique, as it represents the first measurements and vertical survey of the ionospheric population - along the trajectory of Rosetta - near the surface of a cometary nucleus. This paper aims at reporting and explaining the plasma densities observed by the RPC-MIP and RPC-LAP instruments. To interpret the observed plasma densities, we use an ionospheric model in a similar manner as Galand et al. (2016) by using several data set: the ROSINA-COmetary Pressure Sensor (COPS) neutral densities (Balsiger et al. 2007), the RPC-IES (Burch et al. 2007) suprathermal electron fluxes corrected for the spacecraft potential measured by RPC-LAP (Odelstad et al. 2015), and the solar fluxes measured by the Thermosphere Ionosphere Mesosphere Energetics and Dynamics (TIMED)/Solar EUV Experiment (SEE) (Woods et al. 2005). The originality of this study with respect to the one presented in Galand et al. (2016) comes first from the uniqueness of the data set we are assessing (the cometocentric distance ranges down to the surface) and the implementation of a neutral adiabatic model, which is critical to understand the plasma densities close to the surface. This neutral model is recalibrated at each ROSINA-COPS data point to take into account the acceleration of the neutral outflow and remove some of the variability associated with the geometric position of the spacecraft. The model does not take in account any plasma acceleration process due to the ambient electromagnetic field or plasma dynamics but is used to identify when and where these phenomena take place. In other words, we only take in account few (though predominant) physical phenomena but with a data-based accuracy and a high time resolution. Time resolution is critical when assessing a data set coming from a spacecraft during a landing phase, with a highly variable position in the reference frame of the comet. Never during the Rosetta mission, the spacecraft had its cometocentric distance changing at such a fast pace.
Section 2 presents the RPC-MIP and RPC-LAP data set to derive cross-calibration consistency in terms of the electron number density time series during the final descent. A 3D mapping of the descent is provided. The different retrieval approaches for the electron densities are presented and an averaged electron temperature is estimated. Section 3 describes the ionospheric model with its similarities and differences with respect to the one proposed in Galand et al. (2016). Some of the ionospheric density features observed in the RPC-MIP and RPC-LAP data of the descent are derived analytically. Section 4 confronts the model to the RPC data. There is overall excellent agreement between the probed electron densities and the modelled ion densities. It is however interesting to identify the occasional disagreements to understand the physics associated with them.

\section{PLASMA DENSITY PROFILE DURING Rosetta's DESCENT TO THE COMET SURFACE}

The RPC instruments were continuously operating during the descent of Rosetta, until impact on the comet surface at 10:39 UT on 2016 September 30. The Rosetta spacecraft position is shown in Fig. 1, panels (i-iii), in the cometocentric frame. RPC-MIP (Trotignon et al. 2007) was operating in the so-called short Debye length mode, with phased transmitters, to measure mutual impedance spectra with an average cadence of about $5 \mathrm{~s}$. Both RPC-LAP LAngmuir Probes (Eriksson et al. 2007) were operated in floating mode, in which the probe-to-spacecraft potential of each probe was recorded at $4.4 \mathrm{~s}$ time resolution most of the time. We mainly use data from LAP2, located $1.6 \mathrm{~m}$ from the spacecraft body, which was sunlit all the time of interest and therefore provides a more stable reference than LAP1 in this case. Both the RPC-MIP mutual impedance spectra and the RPC-LAP electric potential enable to retrieve the plasma density. The data sets from both instruments have been cross-calibrated to obtain the best possible time resolution and accuracy for the plasma density.

\subsection{RPC-MIP measurements}

The evolution of the plasma line is shown on the RPC-MIP mutual impedance spectrogram in Fig. 1, panel (iv), where each spectrum has been rescaled from its minimum (blue) to its maximum (red) values. Interferences observed since launch appear on top of the signal, at harmonics of 49 and $170 \mathrm{kHz}$. The plasma frequency is extracted, when possible, from the MIP mutual impedance spectra (using both amplitude and phase measurements) and converted into electron density $n_{\text {MIP }}$, shown in Fig. 1, panel (v), for individual spectra (grey dots) and as a moving median (red line) to filter out the density fluctuations associated with high-frequency plasma dynamics. The plasma density increases from 50 to about $150 \mathrm{~cm}^{-3}$ at 09:30 UT, before decreasing as Rosetta finished its descent to the comet surface. Note that mutual impedance experiments require emitters and receivers to be far enough (in terms of the Debye length) to enable to retrieve the plasma frequency and hence the plasma density. This means that mutual impedance experiments are blind below a density threshold, about $50 \mathrm{~cm}^{-3}$ for the operational mode used during end of mission.

\subsection{RPC-LAP measurements}

The evolution of the LAP2 probe-to-spacecraft potential $V_{\mathrm{LAP} 2}$, with the probe floating (zero current between probe and instrument), is shown in Fig. 1, panel (vi). This relates to the spacecraft potential $\phi_{\mathrm{s} / \mathrm{c}}$ as $V_{\mathrm{LAP} 2}=-\alpha \phi_{\mathrm{s} / \mathrm{c}}$, where the correction factor $\alpha$ accounts for 

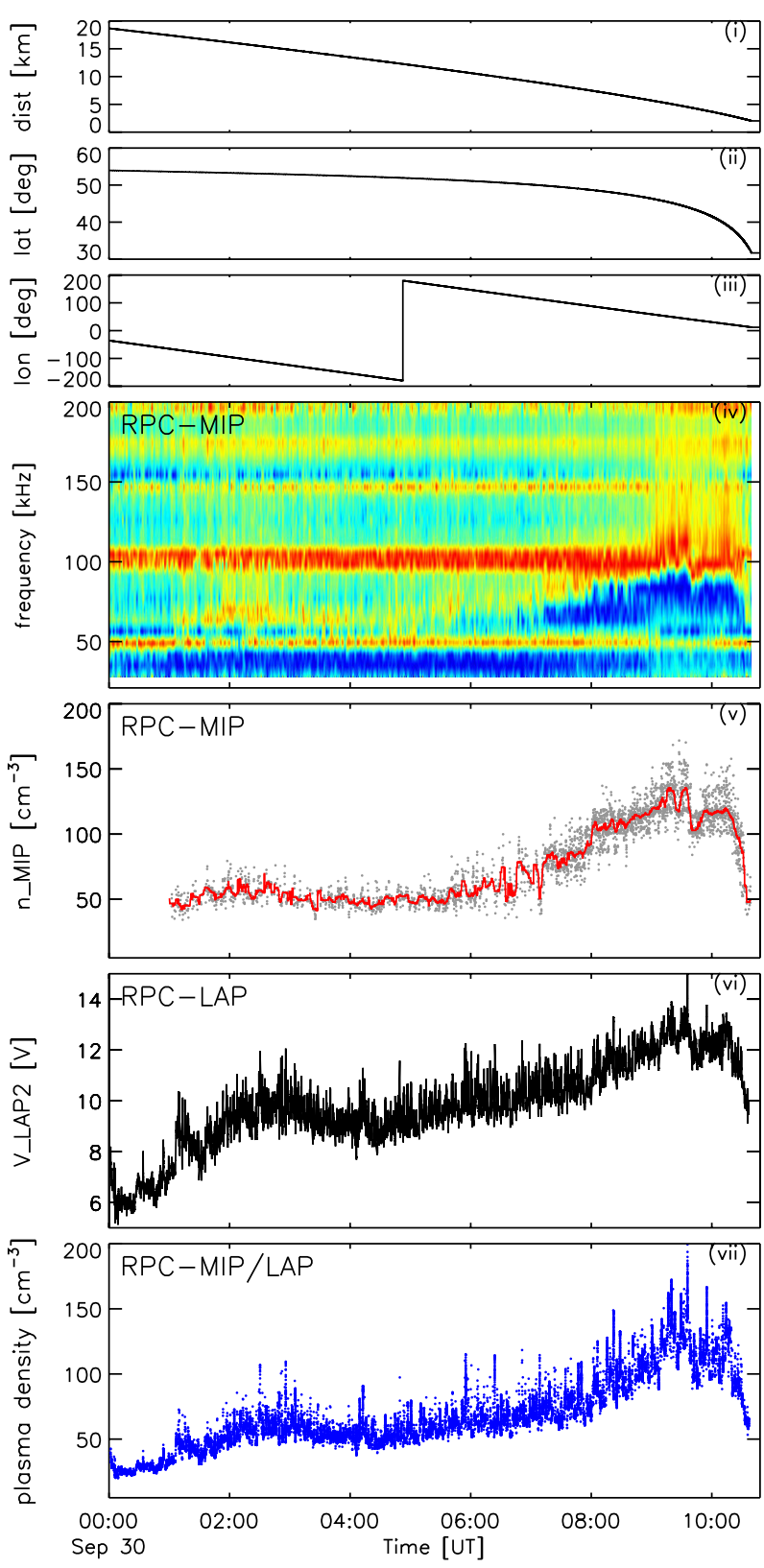

Figure 1. Top to bottom panels: (i) cometocentric distance, (ii) latitude and (iii) longitude of the Rosetta spacecraft during 2016 September 30, (iv) normalized spectrogram of the RPC-MIP mutual impedance amplitude (blue: minimum, red: maximum); (v) RPC-MIP derived electron density for single RPC-MIP mutual impedance spectra (grey dots) and overplotted 5 min moving median (red line); (vi) RPC-LAP2 potential at fixed current bias; (vii) cross-calibrated RPC-MIP/LAP plasma density.

imperfect shielding of the spacecraft potential at the probe position. A typical value for $\alpha$ is 0.8 (Odelstad et al. 2017). For the main use of LAP data in this paper, namely calibrating spacecraft potential variations to plasma density from MIP, we are not sensitive to the exact value of $\alpha$.

In a tenuous plasma, $\phi_{\mathrm{s} / \mathrm{c}}$ is the potential at which the currents to the spacecraft due to impacts of ambient plasma electrons and emission of photoelectrons from sunlit parts of the spacecraft sur- face balance each other. For a negatively charged spacecraft, the photoelectron current is saturated and independent of $\phi_{\mathrm{s} / \mathrm{c}}$. To good approximation, the collection of ambient plasma electrons follows the orbit motion limited theory (Mott-Smith \& Langmuir 1926) and the ambient electron current is thus proportional to the ambient electron density $n_{\mathrm{e}}$ and the exponential of $\phi_{\mathrm{s} / \mathrm{c}}$. This implies that $\phi_{\mathrm{s} / \mathrm{c}}$ is proportional to the logarithm of $n_{\mathrm{e}}$ and, as shown by Odelstad et al. (2015), when $\phi_{\mathrm{s} / \mathrm{c}}$ is known from measurements one can solve for $n_{\mathrm{e}}$, giving

$n_{\mathrm{e}}=\frac{C}{\sqrt{T_{\mathrm{e}}}} \exp \left(\frac{\phi_{\mathrm{s} / \mathrm{c}}}{T_{\mathrm{e}}}\right)$

where $T_{\mathrm{e}}$ is the electron temperature in $\mathrm{eV}$ and $C$ is a proportionality constant taking into account the spacecraft current-collecting and illuminated areas and the solar UV flux at the position of the spacecraft. In this paper, an explicit value of $C$ is not necessary since $n_{\mathrm{e}}$ is obtained from cross-calibration with RPC-MIP.

\subsection{Cross-calibration of RPC-MIP plasma density and RPC-LAP spacecraft potential variations}

For isothermal electrons, the LAP2 floating potential is proportional to the logarithm of the electron density $n_{\mathrm{e}}$. Therefore, to calibrate the LAP2 potential in terms of plasma densities through the MIP absolute plasma density measurements, we fit linearly $V_{\mathrm{LAP} 2}$ to $\log \left(n_{\mathrm{MIP}}\right)$ to retrieve a cross-calibrated MIP-LAP plasma density time series, shown in Fig. 1, panel (vii). We thus make use of the LAP spacecraft floating potential measurements to first validate the MIP-retrieved plasma density when it is above $50 \mathrm{~cm}^{-3}$, and secondly, to extrapolate it before 01:00 UT and after when the plasma density is of the order of or lower than the MIP detectability threshold. On top of that, the fit provides an estimate of the mean electron temperature during 2016 September $30, T_{\mathrm{e}} / \alpha \simeq 3.44 \pm 0.51 \mathrm{eV}$, consistent with what has been observed by LAP Langmuir probe sweeps throughout the mission during the low-activity phase of the comet. With an $\alpha$ value of 0.8 (Section 2.2), this gives an average electron temperature $T_{\mathrm{e}} \simeq 2.75 \pm 0.41 \mathrm{eV}$ (at a 95 per cent confidence level).

The plasma density vertical profile observed from $20 \mathrm{~km}$ down to the comet surface on 2016 September 30 (Rosetta's end of mission descent) is shown in Fig. 2. Close to the comet surface, the cometary ionospheric density increases with cometocentric distance, until it reaches a maximum around $5 \mathrm{~km}$ (corresponding to about $3 \mathrm{~km}$ altitude). Above this value, the cometary ionospheric density decreases roughly as the inverse of the cometocentric distance $r$ (blue lines in Fig. 2), consistent with what has been observed in 2015 February (Edberg et al. 2015). The $r^{-1}$ representation ceases to match the observation when the cometocentric distances reach the same order of magnitude $(<6 \mathrm{~km})$ as the nucleus radius $r_{0}$ $(2 \mathrm{~km})$. In fact, the cometocentric distance dependence of the ionospheric density is $\left(r-r_{0}\right) / r^{2}$ under constant outflow velocity (see Section 3.1).

The cometary ionospheric density is also shown along the spacecraft trajectory in the fixed comet frame in Fig. 3 for which a movie is made available within the online supplementary material. Note that the trajectory is associated with large variations in latitude/longitude during the descent, so that the vertical profile shown in Fig. 2 may still be influenced by local variations in the coma, as for instance at 03:00 uT while Rosetta was located at $15 \mathrm{~km}$ from the comet, above the illuminated neck (see Section 3.3). 


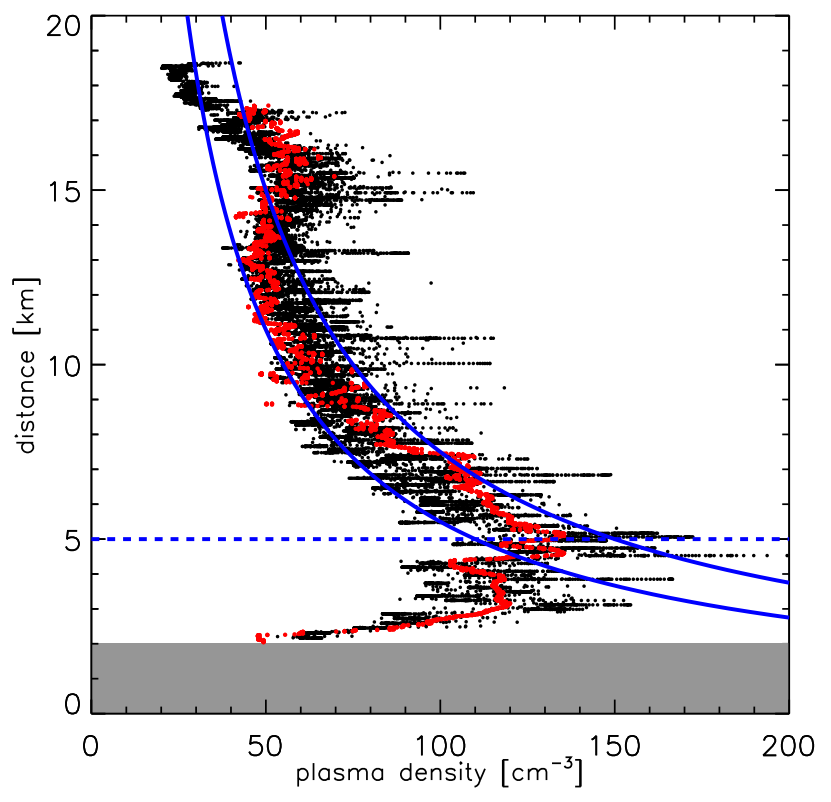

Figure 2. Vertical profile of the cross-calibrated plasma density (red dots: MIP density, black dots: cross-calibrated MIP-LAP density) along the trajectory of Rosetta on 2016 September 30 . Blue lines show $1 / r$ density variation profiles. The blue dotted line indicates the location of the maximum density profile. The grey region indicates the comet subsurface region at the impact position.

\section{IONOSPHERIC MODEL}

\subsection{Model description}

The ionospheric model is based on a 1D continuity equation applied to the entire ionospheric population under spherical symmetry assumption:

$\frac{\partial n_{j}(r, t)}{\partial t}+\frac{1}{r^{2}} \frac{\partial}{\partial r}\left(r^{2} u_{j}(r) n_{i}(r, t)\right)=S_{j}(r, t)$,

where $n_{j}$ stands for the number density of ion species $j, u_{j}$ stands for the bulk velocity of the same ion species and $S_{j}(r, t)$ stands for its net production. This last term regroups production through photoionization, electron-impact ionization, charge exchange with solar wind ions, ion-neutral chemical reactions, and loss through electron-ion dissociative reactions or ion-neutral chemistry.

Time convergence is reached quickly by comparison to the timescale of ion production at low cometocentric distances (Galand et al. 2016). We therefore assume steady state (i.e. $\partial / \partial t=0$ ). We also assume no acceleration of the ions through external fields. This assumption is only valid close to the comet and is discussed in Section 3.5. Under this specific assumption, the ions keep, once produced, the same bulk velocity as the neutrals, which undergo acceleration themselves (i.e. $u_{i}=u$ for all $i$ ). Finally, we assume no electron-dissociative recombination as it is weak for low-activity comets. Validity of this last assumption is discussed in details by Galand et al. (2016).

We sum equation (1) over all the ion species $j$. The ion-neutral chemistry gain and loss terms (e.g. $\mathrm{H}_{2} \mathrm{O}+\mathrm{H}_{2} \mathrm{O}^{+} \rightarrow \mathrm{OH}+\mathrm{H}_{3} \mathrm{O}^{+}$) on the right-hand side cancel each other to leave only the total ion production terms through ionization (Galand et al. 2016):

$\frac{1}{r^{2}} \frac{\mathrm{d}}{\mathrm{d} r}\left(r^{2} u(r) n_{i}(r)\right)=\left(v^{\mathrm{e}-}(r)+v^{h v}(r)\right) n(r)$,

\section{MIP-LAP cross-calibrated plasma density [ $\mathrm{cm}-3]$}
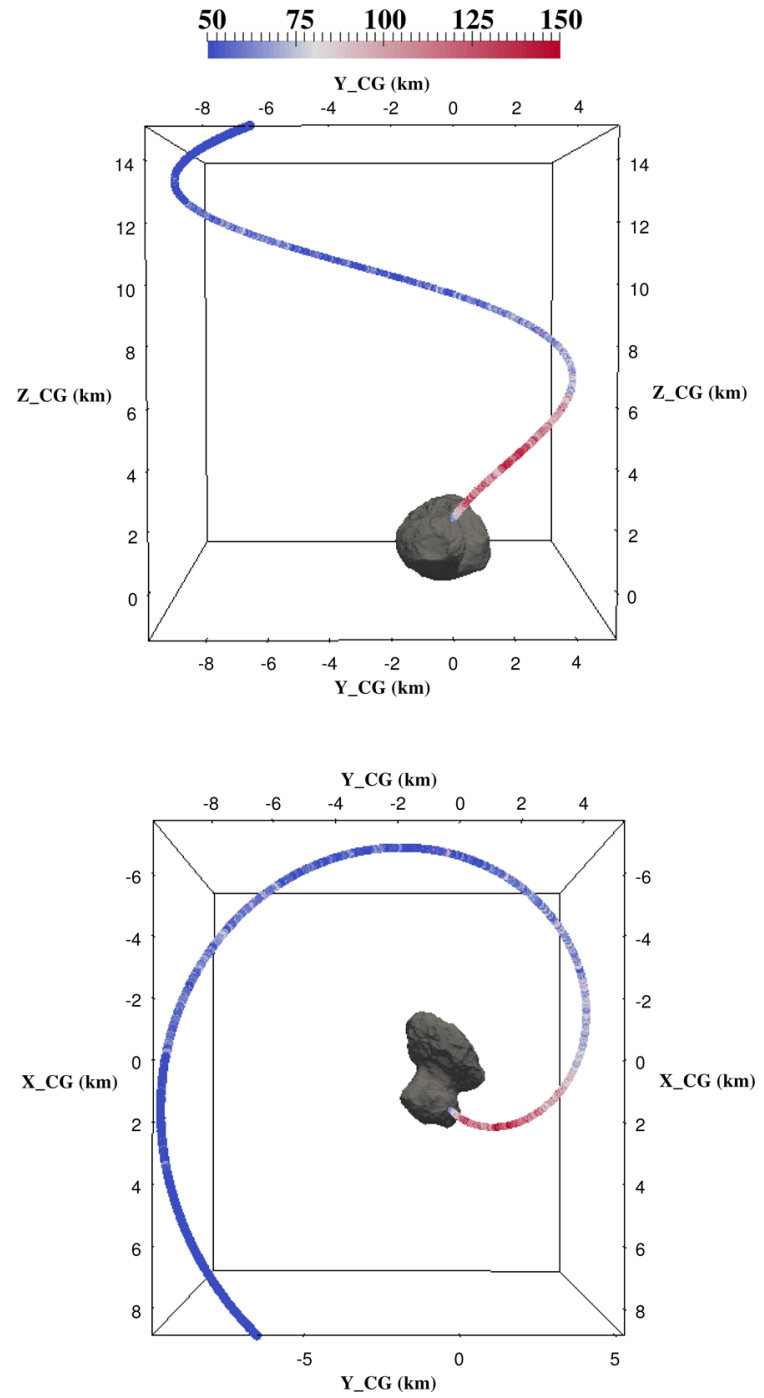

Figure 3. Plasma density along the spacecraft trajectory in the comet fixed frame $\left(Z_{C G}\right.$ is the rotation axis) on 2016 September 30 . Full movie available within the supplementary online material.

where $n_{i}$ stands for the total ion number density $\left(n_{i}=\sum_{j} n_{j}\right)$ and $n$ stands for the total neutral number density. The frequencies $v^{\mathrm{e}-}(r)$ and $v^{h v}(r)$ stand for the electron-impact ionization and photoionization rates, respectively. Charge-exchange between solar wind ions and cometary neutrals is not included in the model but is discussed in Section 3.4.3. We consider a full $\mathrm{H}_{2} \mathrm{O}$ mixture. In general, it is a good assumption even with an important quantity of $\mathrm{CO}_{2}$ in the coma. Indeed, even though the ionization cross-sections of $\mathrm{CO}_{2}$ are higher than the ones of $\mathrm{H}_{2} \mathrm{O}$, this effect is counter balanced by the lower sensitivity of COPS with respect to $\mathrm{CO}_{2}$ (Gasc et al. 2017). Because of this factor, even if $v$ increases with the presence of $\mathrm{CO}_{2}$, the increase of the product $v \times n$ is moderated by the correction for neutral composition. Galand et al. (2016) did a full sensitivity study on this effect. By taking into account the correction factor of COPS, the photoionization frequency is only increased by a factor 1.18 at most from a pure $\mathrm{H}_{2} \mathrm{O}$ to a half $\mathrm{CO}_{2}$ half $\mathrm{H}_{2} \mathrm{O}$ mixture, whereas the electron-impact ionization frequency is increased by about 1.14 or less. Therefore, a model that focuses on the total number of ions 
without assessing the ion composition does not need an accurate input of the neutral mixing ratios to compute a good estimation.

The photoionization frequencies are estimated from TIMED/SEE integrated solar flux (Woods et al. 2005) and the electron-impact ionization from RPC-IES (Burch et al. 2007) instantaneous suprathermal electron flux density (see Section 3.4). In order to integrate (2), a further assumption is that $v^{h v}(r)$ and $v^{\mathrm{e}-}(r)$ are independent of $r$. This is a good approximation when the neutral density is low enough. By solving the Beer-Lamber law, we found that the attenuation of EUV solar radiation, and hence the photoionization rate, through solar photon absorption in the coma was not significant at the end of mission. It is attenuated by less than 14 per cent at the surface and less than 2 per cent at a cometocentric distance of $10 \mathrm{~km}$. This assumption is not valid during the whole mission, especially around perihelion, where the photoionization rate is attenuated by over 90 per cent at the surface (Heritier et al. 2017). As for the electron-impact ionization rate, the neutral population is not dense enough at high heliocentric distances to drive significant energy degradation of suprathermal electron flux (Galand et al. 2016). Equation (2) can thus be integrated to

$n_{i}(r)=\frac{v^{\mathrm{e}-}+v^{h v}}{r^{2} u(r)} \int_{r_{0}}^{r} n\left(r^{\prime}\right) r^{\prime 2} \mathrm{~d} r^{\prime}$,

where $r_{0}$ stands for the average nucleus radius $(2 \mathrm{~km})$. In Section 4 , we use two different approaches to compute the integral on the right-hand side of this equation. The first approach is an analytical solution in the specific case of the neutral number density following Haser's model with a constant outflow velocity. The neutral number density thus solely varies with $r^{-2}$ and the integral is computed analytically (Galand et al. 2016):

$n_{i}(r)=\left(v^{\mathrm{e}-}+v^{h v}\right) \frac{r-r_{0}}{u} n(r)$.

This simplified model exhibits good agreements with the RPC-MIP and RPC-LAP probed electron number densities at cometocentric distances of $10-20 \mathrm{~km}$ over the 2014 October period (3.15 au from the Sun) as shown by Galand et al. (2016). As $n(r)$ is solely a function of $r^{-2}, n_{i}(r)$ is ultimately proportional to $\left(r-r_{0}\right) / r^{2}$, which can be assimilated with a $r^{-1}$ trend for a fixed production rate and at sufficiently high cometocentric distances (when $r$ is high with respect to $r_{0}$ ). This $r^{-1}$ trend was indeed noticed in Section 2.3.

The second approach adopted in this paper takes into account the increase of the neutral outflow velocity as the gas moves away from the nucleus. In that case, the integral of equation (3) is computed numerically. The column density $n\left(r^{\prime}\right)$ for $r^{\prime}=r_{0}$ to $r$ and the neutral bulk velocity $u(r)$ are computed through an adiabatic coma expansion model driven by ROSINA-COPS. The model and its conditions of validity are explained in details in Heritier et al. (2017) and reviewed in Section 3.3.

\subsection{Location of the ionosphere density peak}

In Section 2.3, we highlighted the presence of a peak in ionospheric densities at about $5 \mathrm{~km}$ from the centre of the nucleus. This peak was predicted by Vigren \& Galand (2013). One can wonder if it is possible to derive, from the equations given in Section 3.1, the location of the peak. We assume neutral distribution proportional to the inverse square of the cometocentric distance $\left(n(r) \sim r^{-2}\right)$, such that $n(r)=n\left(r_{1}\right) r_{1}^{2} / r^{2}$, where $r_{1}$ is a fixed cometocentric distance. By taking the derivative of equation (4), we find

$\frac{\mathrm{d} n_{i}}{\mathrm{~d} r}(r)=\left(v^{\mathrm{e}-}+v^{h v}\right) \frac{n\left(r_{1}\right) r_{1}^{2}}{u} \times \frac{2 r_{0}-r}{r^{3}}$.

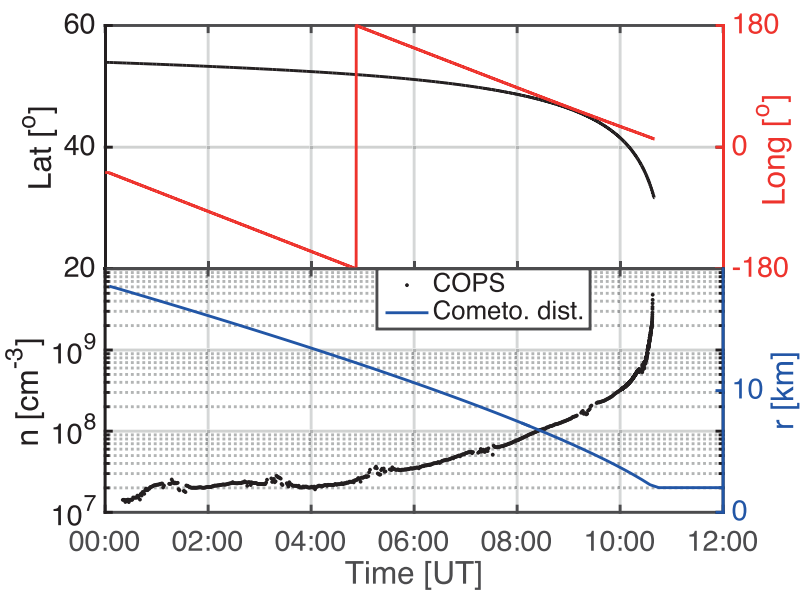

Figure 4. Top: Time series of the spacecraft latitude (black line) and spacecraft longitude (red line). Bottom: Time series ROSINA-COPS neutral number density (black dots) and the cometocentric distance (blue lines).

The expression (5) cancels for $r=2 r_{0}$, where $r_{0}$ is the theoretical radius of comet $67 \mathrm{P}$ (typically $2 \mathrm{~km}$ for comet $67 \mathrm{P}$ ). Physically, we can guess that it corresponds to a maximum but we can verify mathematically that the second derivative is negative for $r=2 r_{0}$.

$\frac{\mathrm{d}^{2} n_{i}}{\mathrm{~d} r^{2}}\left(2 r_{0}\right)=-\frac{1}{\left(2 r_{0}\right)^{3}} \times\left(v^{\mathrm{e}-}+v^{h v}\right) \frac{n\left(r_{1}\right) r_{1}^{2}}{u}<0$.

The maximum plasma density occurs at a cometocentric distance equal to twice the theoretical radius of the nucleus, with a magnitude of $\left(v^{\mathrm{e}-}+v^{h v}\right) n\left(2 r_{0}\right) r_{0} / u$ (from equation 4$)$. Note that the location of the peak only depends on the geometry of the nucleus. It is independent of the magnitude of the different source terms, neutral density or outflow bulk velocity. The RPC-MIP and RPC-LAP measurements recorded a maximum electron density at around $5 \mathrm{~km}$ which is quite close to the theoretical value of $2 r_{0}=4 \mathrm{~km}$, being given the uncertainty in $r_{0}$ and the non-spherical shape of the comet. Some of the assumptions made may drive a departure from this value, such as a $r$-dependent outflow velocity (as it is considered in the adiabatic neutral model, see Section 4.2), $r$-dependent ionization rates as well as a non-spherical nucleus and a non-uniform outgassing rate.

\subsection{Neutral coma}

To run the ionospheric model and solve equation (3), it is necessary to know the neutral background conditions. Fig. 4 shows the time series of the spacecraft latitude (top, black) and longitude (top, red), ROSINA-COPS (Balsiger et al. 2007) neutral number densities (bottom, black) within a margin of error of \pm 15 per cent and cometocentric distance (bottom, blue) during the final descent.

The activity of the comet was overall very low due to the high heliocentric distance (3.8 au). Indeed, for an average speed of $500 \mathrm{~m} \mathrm{~s}^{-1}$, the Haser model $\left(n=Q /\left(4 \pi u r^{2}\right)\right)$ provides an outgassing rate $Q$ of the order of $4 \times 10^{25} \mathrm{~s}^{-1}$. It is quite low compared to the outgassing of $4 \times 10^{28} \mathrm{~s}^{-1}$ near perihelion (Hansen et al. 2016). If we compare over the whole mission, the last time the activity was this weak was in 2014 September, shortly after the rendezvous of Rosetta with 67P. However, at the end of mission, the observed neutral number densities were extremely high due to the proximity of the spacecraft to the nucleus. The observed densities just before the impact of the spacecraft are about $5 \times 10^{9} \mathrm{~cm}^{-3}$. It is even higher than the densities of $10^{8} \mathrm{~cm}^{-3}$ measured by ROSINA-COPS near 

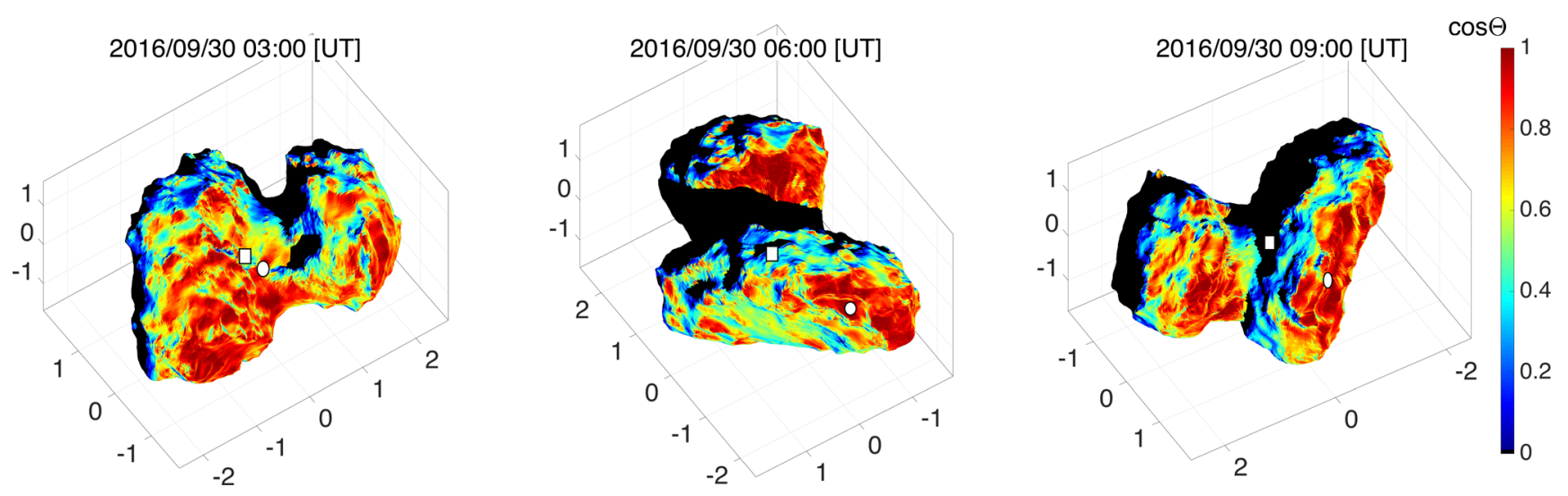

Figure 5. Illumination model from Rosetta's point of view at three given times during the final descent on 2016 September 30 . The axis scale is in kilometres. The colour code corresponds to the cosine of the illumination angle (i.e. the angle between the normal of a face and the Comet-Sun axis). The white square corresponds to the sub-spacecraft point and the white circle corresponds to the sub-solar point. At 3:00 and 9:00 UT, the neck of the nucleus is illuminated and visible from the spacecraft. The distance between the spacecraft and the nucleus is not represented in this plot. A full movie is available within the supplementary online material.

perihelion (Fougere et al. 2016) as the spacecraft trajectory adopted a safety distance of at least $160 \mathrm{~km}$ over this period.

The longitude and latitude time series show that the angular positions of the spacecraft in the comet reference frame were highly variable. It is clearly visible on the $3 \mathrm{D}$ plot in Fig. 5, which shows the nucleus from the point of view of the spacecraft and for which a full movie is available within the online supplementary material. The integral in equation (3) requires the sub-spacecraft neutral number density $n$ as a function of the cometocentric distance $r$, whereas the data set of Fig. 4 only gives the in situ neutral density at the location of Rosetta. It is not recommended to directly use a pseudo-derived data set of $n$ as a function of $r$ from Fig. 4 to provide the subspacecraft column density as the activity varies for different latitudes and longitudes in the comet reference frame. Indeed, the neutral density profile below the spacecraft changes due to diurnal and seasonal dependencies of the outgassing rate (Hässig et al. 2015), its topography (Biver et al. 2015) and the illumination conditions (Bieler et al. 2015; Mall et al. 2016) depicted in Fig. 5 for the end of mission. Instead, a neutral model described in Heritier et al. (2017) is calibrated to the COPS measured density at the location of the spacecraft and its respective column of density is numerically estimated at a given time. This process is iterated for each COPS data point. Profiles in neutral density and bulk velocity (used in Section 4.2) are computed along the spacecraft-nucleus line at each time step.

\subsection{Ionizing sources}

\subsubsection{Photoionization}

Photoionization is computed with TIMED/SEE (Woods et al. 2005) measurement of the solar flux spectral flux $F_{1 \text { au }}(\lambda)$. These observations are made from Earth orbit. They are then adjusted to exactly 1 au such that it is easily computable for other heliocentric distances. The corresponding photoionization frequency at 1 au can be computed through

$v_{1 \mathrm{au}}^{h v}=\int_{\lambda_{\min }}^{\lambda^{t h}} \sigma_{i o n i}^{h v}(\lambda) F_{1 \text { au }}(\lambda) \mathrm{d} \lambda$,

where $\sigma_{\text {ioni }}^{h v}(\lambda)$ stands for the $\mathrm{H}_{2} \mathrm{O}$ photoionization cross-section taken from Vigren \& Galand (2013), $\lambda^{\text {th }}$ corresponds to the

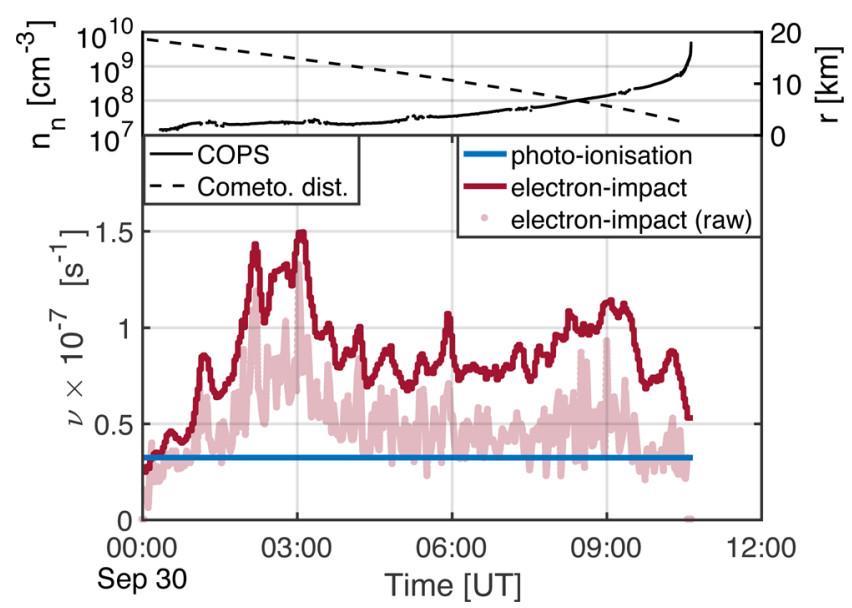

Figure 6. Top: Time series of the COPS neutral number density (full line) and cometocentric distance (dashed line). Bottom: Time series of the effective photoionization frequency $v_{3.8 \text { au }}^{h v}$ (blue curve), electron-impact ionization frequencies corrected with the spacecraft potential (red curve) and uncorrected (pink dots). Pure water has been assumed for the neutral composition.

maximum wavelength below which absorption of solar photons possibly leads to ionization, $\lambda_{\min }$ (here $0.1 \mathrm{~nm}$ ) is a wavelength below which $\sigma_{n}^{h v \text {,ioni }}(\lambda)$ and $F_{1 \text { au }}(\lambda)$ drop drastically.

On 2016 September 30, the heliocentric distance of comet 67P was 3.8 au. The decrease in photoionization frequency is dictated by

$v_{3.8 \mathrm{au}}^{h v}=\frac{v_{1 \mathrm{au}}^{h v}}{d_{\mathrm{au}}^{2}}=\frac{v_{1 \mathrm{au}}^{h v}}{3.8^{2}}$.

The time series of the effective photoionization frequency, corrected for heliocentric distance, are plotted in Fig. 6. The time resolution of TIMED/SEE is $1 \mathrm{~d}$ and remains thus constant in this plot. The solar spectrum used was sampled on 2016 October 12 . Indeed, due to a phase shift of $-167^{\circ}$ between comet $67 \mathrm{P}$ and the Earth, the same face of the Sun that was facing the Earth on October 12 was facing the comet on September 30. Overall, the photoionization rate is relatively low compared to the rest of the mission because of the high heliocentric distance and the decreasing solar activity. The 
number of sunspots is subject to an $11 \mathrm{yr}$ period and has been overall decreasing since Rosetta arrived at comet 67P in 2014 August.

\subsubsection{Electron-impact ionization}

The electron-impact ionization frequency is estimated from in situ measurement of RPC-IES (Burch et al. 2007). We need however to make assumptions for deriving the electron-impact ionization frequency $v^{\mathrm{e}-}$ at lower cometocentric distances. On 2016 September 30 , the spacecraft was located very close from the nucleus $(r \leq$ $20 \mathrm{~km}$ ), and the comet was weakly active (see Section 3.3). It is therefore legitimate to assume that the electron flux is not too disturbed by the cometary environment (no energy degradation above the $\mathrm{H}_{2} \mathrm{O}$ ionizing energy threshold $\sim 12.6 \mathrm{eV}$ ) and relatively constant in the neighbourhood of Rosetta (Galand et al. 2016). We have therefore assumed that $v^{\mathrm{e}-}$ is independent of $r$, which is not necessarily valid at other periods of the mission such as perihelion.

The $\mathrm{H}_{2} \mathrm{O}$ electron-impact ionization frequency is computed from the RPC-IES measured electron flux density $J_{\mathrm{e}}(E)$ through

$v^{\mathrm{e}-}=\int_{E^{\text {th }}}^{E_{\max }} \sigma_{\text {ioni }}^{\mathrm{e}-}(E) J_{\mathrm{e}}(E) \mathrm{d} E$,

where $\sigma_{\text {ioni }}^{\mathrm{e}-}(E)$ stands for the ionization cross-section of $\mathrm{H}_{2} \mathrm{O}$ taken from Vigren $\&$ Galand (2013), $E^{\text {th }}$ corresponds to the minimum energy above which electron impacts can lead to ionization of water (12.6 eV from Itikawa \& Mason 2005) and $E_{\max }$ is taken as $200 \mathrm{eV}$. Above this energy, the suprathermal electron flux is too weak to be a significant ionizer. Most of the electron-impact ionization happens in the $20-100 \mathrm{eV}$ range (e.g. Galand et al. 2016). RPC-IES electron flux densities are retrieved from counts by using the in-flight calibrated geometric factor and the MCP efficiencies applied to each energy bin. The counts are then integrated along azimuthal and elevation angles. We assume isotropy of the electron population to estimate the fluxes in the missing field of view.

The negatively charged spacecraft affects the electron flux density measured by IES. Liouville's theorem implies that the quantity $J_{\mathrm{e}}(E) / E$ is conserved under spherical symmetry (Galand et al. 2016). It is therefore possible to partially retrieve the original electron spectral flux from the spacecraft measured by RPC-LAP through the equation

$J_{\mathrm{e}}(E)=\frac{E}{E_{\mathrm{IES}}} J_{\mathrm{e}}^{\mathrm{IES}}\left(E_{\mathrm{IES}}\right)$,

where $J_{\mathrm{e}}(E)$ represents the actual electron flux density as a function of $E$. $E_{\mathrm{IES}}$ and $J_{\mathrm{e}}^{\mathrm{IES}}\left(E_{\mathrm{IES}}\right)$ represent the energy of the electrons and the electron flux density, respectively, as measured by IES. It is altered by the spacecraft potential such that $E_{\mathrm{IES}}=E+V_{\mathrm{SC}}$, where $V_{\mathrm{SC}}$ is the spacecraft potential measured by LAP (negative quantity). The flux can only be partially restored because low-energy electrons with $E<\left|V_{\mathrm{SC}}\right|$ are repelled before being able to reach the spacecraft and the instrument. However, since the absolute value of the spacecraft potential in this period was always lower than the ionization energy threshold used in equation (9) (i.e. $\left|V_{\mathrm{SC}}\right|<E^{\text {th }}$ at any time), the missing part of the distribution is not relevant to our calculation.

The time series of the electron-impact ionization frequency are plotted in Fig. 6. The red curve corresponds to the electronimpact frequency corrected with the RPC-LAP spacecraft potential, whereas the pink dots correspond to the electron-impact frequency (raw) calculated without correction. The corrected series are more continuous with less variability in time. It shows to what extent the spacecraft potential correction actually restores the original data set as the time-continuous flux is more physical and consistent. No smoothing function was applied between the raw values in pink and the corrected rates in red. What could appear as noise in the raw data is actually induced by the spacecraft potential. As expected, the corrected frequencies are higher than the raw ones, because they integrate the low-energy part of the distribution (10-20 eV). Electrons in this energy range are very efficient in ionizing neutrals. The time resolution of RPC-IES under the mode used on that day is $128 \mathrm{~s}$. We see important variations of the flux measured by RPC-IES down to the surface of comet $67 \mathrm{P}$ as the frequencies vary from $2 \times 10^{-8}$ to $1.5 \times 10^{-7} \mathrm{~s}^{-1}$. Overall, electron-impact ionization dominates over photoionization (see Fig. 6). The model allows us to disentangle the contribution from different sources to the RPC-MIP and RPC-LAP electron densities.

\subsubsection{Charge-exchange with the solar wind}

Another source of ions, not included in the model presented in Section 3.1, is charge-exchange. At high heliocentric distances ( $>2.5 \mathrm{au}$ ), solar wind ions have access to the coma, down to the surface of the nucleus (Wurz et al. 2015; Nilsson et al. 2017). These particles, such as protons, are thus able to exchange a charge with cometary neutrals. For water, the following reaction occurs:

$\mathrm{H}_{\mathrm{sw}}^{+}+\mathrm{H}_{2} \mathrm{O} \rightarrow \mathrm{H}_{2} \mathrm{O}^{+}+\mathrm{H}_{(\mathrm{sw})}$,

where $\mathrm{H}_{\mathrm{sw}}^{+}$stands for an incoming solar wind proton. This reaction does not produce an electron. The neutral hydrogen $\mathrm{H}_{(\mathrm{sw})}$ keeps most of its energy $(\sim 1 \mathrm{keV})$ in the process and is therefore more energetic than the surrounding neutral population. Other solar wind ions interact with the cometary neutrals, such as the alpha particle $\mathrm{He}^{2+}$ (Goldstein et al. 2015; Nilsson et al. 2015a) but the main charge exchange source remains through protons that constitute about 92 per cent of the solar wind population (Cravens 1997).

During the Rosetta escort phase, RPC-ICA (Nilsson et al. 2007) monitored the solar wind fluxes. By extracting the solar wind proton flux, we are able to compute the first and second moments of the proton distribution function to estimate the solar wind density $n^{\mathrm{sw}}\left(\mathrm{H}^{+}\right)$ $\left(0.34 \pm 0.24 \mathrm{~cm}^{-3}\right)$ and bulk velocity $u_{\mathrm{H}^{+}}^{\mathrm{sw}}\left(519 \pm 54 \mathrm{~km} \mathrm{~s}^{-1}\right)$. The charge exchange cross-section for protons with $\mathrm{H}_{2} \mathrm{O}$ depends on the bulk speed through the following equation (Tawara 1978; Rubin et al. 2009):

$\sigma^{X}\left(\mathrm{H}^{+}, \mathrm{H}_{2} \mathrm{O}\right)=\left(5.93-0.174 \log \left(u_{\mathrm{H}^{+}}^{\mathrm{sw}}\right)\right)^{2} \times 10^{-16}\left(\mathrm{~cm}^{2}\right)$,

where $u^{\mathrm{sw}}$ is expressed in $\mathrm{cm} \mathrm{s}^{-1}$ to get the resulting cross-section in $\mathrm{cm}^{2}$.

We have computed the average value of $\left\langle n^{\mathrm{sw}}\left(\mathrm{H}^{+}\right) u_{\mathrm{H}^{+}}^{\mathrm{sw}}\right.$ $\left.\sigma^{X}\left(\mathrm{H}^{+}, \mathrm{H}_{2} \mathrm{O}\right)\right\rangle$ measured by RPC-ICA during the descent. We get an estimate of the charge-exchange frequency of about $3.84 \mathrm{~s}^{-1}$. It is the same order of magnitude as the photoionization frequency (see Fig. 6). In Section 4, we compare the results of the ionospheric model with the total measured electron population. We are currently unable to compute an accurate charge-exchange frequency time series and its implementation to our model would require a multidimensional treatment. As our estimated charge-exchange average frequency is significantly lower than the electron-impact ionization frequency, we have neglected it in our model.

\subsection{Plasma exobase}

In this section, we discuss the assumption that the ions moving radially at the same velocity as the neutrals and not undergoing other acceleration processes between the cometary surface and the 


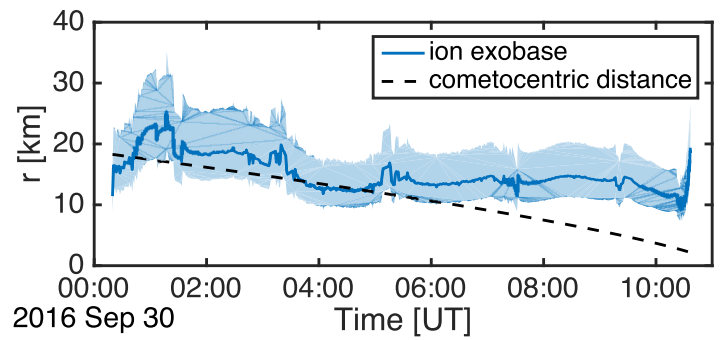

Figure 7. Time series of the computed cometocentric distance of the ion exobase (blue) and Rosetta cometocentric distance (black dashed line) at the end of mission. In the light blue area, the central blue curve corresponds to an ion energy of $0.070 \mathrm{eV}$ whereas the upper bound and lower bound correspond to ion energy of 0.050 and $0.10 \mathrm{eV}$, respectively.

spacecraft. When neutrals are photoionized, the photon energy minus the ionization threshold energy - goes to the newborn photoelectron. Ions have therefore a low energy at their birth ranging from about $0.015 \mathrm{eV}\left(400 \mathrm{~m} \mathrm{~s}^{-1}\right)$ to $0.070 \mathrm{eV}\left(800 \mathrm{~m} \mathrm{~s}^{-1}\right)$. Within the coma, the external field can accelerate these ions. However, at $20 \mathrm{~km}$, considering a typical solar wind magnetic field of $1 \mathrm{nT}$ at $3 \mathrm{au}$, the propagation time from the surface to the spacecraft is at least 25 times less than the gyro-period of water ions (Fuselier et al. 2015). There is time for some acceleration, but not enough to reach values anywhere near the solar wind velocity.

The extra energy gained via external forces, such as the motional electric field, can be lost through ion-neutral collisions as long as the ions are located below the ion exobase (Lemaire \& Scherer 1974). The ion exobase corresponds to the cometocentric distance at which the Knudsen number for the ion species is equal to 1 . This Knudsen number can be expressed as the ratio between the ion mean free path $\lambda$ and the characteristic length $H$. The characteristic length is generally defined as

$H=\frac{n_{i}}{\left|\nabla n_{i}\right|}$.

These quantities are assessed using the refined ionospheric model calibrated with ROSINA-COPS and with varying neutral outflow velocity. A more basic approach consists in using the Haser's model with constant outflow velocity $\left(n(r)=Q / 4 \pi u r^{2}\right)$. In that case, $H$ $\simeq r$ for ions, where $r$ is simply the cometocentric distance. This is similar to taking Whipple \& Huebner (1976) collision zone applied to ions from the surface to $r_{\text {coll }}=Q \sigma /(4 \pi u)$. We compared the two approaches and found similar results within 20 per cent error. We used the refined approach in our calculations of the ion exobase shown in Fig. 7.

The mean free path $\lambda=(n \sigma)^{-1}$ was computed using ion-neutral cross-sections $\sigma$ from Fleshman et al. (2012). These cross-sections depend on the energy of the ions in $\mathrm{eV}$. We expect the ions to have an energy of about $0.070 \mathrm{eV}\left(\sigma=4 \times 10^{-14} \mathrm{~cm}^{-2}\right)$ which corresponds to the terminal bulk velocity of the neutrals. However, by anticipating for potential cooling (through collisions with neutrals) or beginning of acceleration via external fields, we considered the $0.05 \mathrm{eV}\left(\sigma=5.3 \times 10^{-14} \mathrm{~cm}^{-2}\right)$ to $0.10 \mathrm{eV}\left(\sigma=2.9 \times 10^{-14} \mathrm{~cm}^{-2}\right)$ range. Under these assumptions, the cometocentric distance of the ion exobase is plotted in Fig. 7 in blue. The central curve corresponds to an ion energy of $0.070 \mathrm{eV}\left(800 \mathrm{~m} \mathrm{~s}^{-1}\right)$. The lower bound and upper bound of the blue area correspond to an energy of $0.10 \mathrm{eV}$ $\left(\sim 1000 \mathrm{~m} \mathrm{~s}^{-1}\right)$ and $0.05 \mathrm{eV}\left(400 \mathrm{~m} \mathrm{~s}^{-1}\right)$, respectively. The cometocentric distance of Rosetta is represented by the black, dashed line. Rosetta is located below the ion exobase throughout most of its descent trajectory. It means that some collisions take place between the ions and the neutrals and any ion acceleration should be partially attenuated.

The electron exobase was assessed using the same approach as for ions. Cross-sections between electrons and neutrals are taken from Itikawa \& Mason (2005). The average electron energy is assumed to be $2.75 \mathrm{eV}$ as assessed by RPC-MIP and RPCLAP cross-calibration (see Section 2.2). The electron-neutral momentum-transfer cross-section is about $4 \times 10^{-16} \mathrm{~cm}^{2}$ and the electron-neutral vibrational excitation is found to be about $2 \times$ $10^{-16} \mathrm{~cm}^{2}$. The corresponding Knudsen number for the electrons is found to be already superior to 5 at the surface of the nucleus (and increasing at higher cometocentric distances). It means that electronneutral collisions rarely happen. This argument is strengthened by the presence of the solar wind driven magnetic field at the surface of the nucleus (Auster et al. 2015). Henri et al. (2017) showed that the electron exobase (located above the surface near perihelion) is directly correlated to the scaleheight of the diamagnetic cavity. Witnessing the magnetic field near the surface implies that the neutrals are not strongly interacting with the bulk of the electron population. The electron flow moves freely into the plasma mixture. However, charge neutrality is ensured by an ambipolar electric field reacting from any departure from the electrons (Cravens 1997).

\section{COMPARISON BETWEEN OBSERVED AND MODELLED ELECTRON DENSITIES}

Using the ionospheric model presented in Section 3, we estimated the ionospheric densities at the location of Rosetta and we compared them to what was measured by RPC-MIP, cross-calibrated with RPC-LAP (see Section 2).

\subsection{Ionospheric model using constant neutral/ion velocity}

The first ionospheric model that is applied adopts the same approach as Galand et al. (2016), described by equation (4), assuming a constant outflow velocity for the ions and their parent neutrals. Fig. 8 shows the estimated ionospheric population using this model through photoionization only (blue area), and using both photoionization and electron-impact ionization (red area). The results of the model are depicted through surface areas to illustrate the uncertainty in the outflow velocity. The upper bound of each area corresponds to the slow flow case of $400 \mathrm{~m} \mathrm{~s}^{-1}$ and the lower bound corresponds to the fast flow case of $800 \mathrm{~m} \mathrm{~s}^{-1}$ along the whole column from the surface to the spacecraft. These velocities are consistent with MIRO observation from the 2014 August (3.6 au) period where Gulkis et al. (2015) and Lee et al. (2015) derived terminal values for $u$ between 600 and $800 \mathrm{~m} \mathrm{~s}^{-1}$ at the subsolar nadir point. Here the solar zenith angle was about $40^{\circ}$. The model is subject to direct inputs of the neutral densities (equation 4) measured by ROSINACOPS (solid, black line) and the cometocentric distance (dashed, black line), shown in the upper panel of Fig. 8. Small interruptions of the ROSINA-COPS time series can be observed occasionally (e.g. 01:10 and 01:30 UT). When it happens, the model uses linear interpolation of the neutral density to compute the ionospheric densities. Results are confronted with the electron number densities measured by RPC-MIP (purple dots, Section 2.1) and RPC-MIP/LAP (pink dots, Section 2.3).

Photoionization alone cannot explain the observed electron densities and electron-impact ionization needs to be included in order to explain the results. This finding was similar to what Galand et al. (2016) found for the 2014 October period over the winter (southern then, northern here) hemisphere. The significant contribution of 


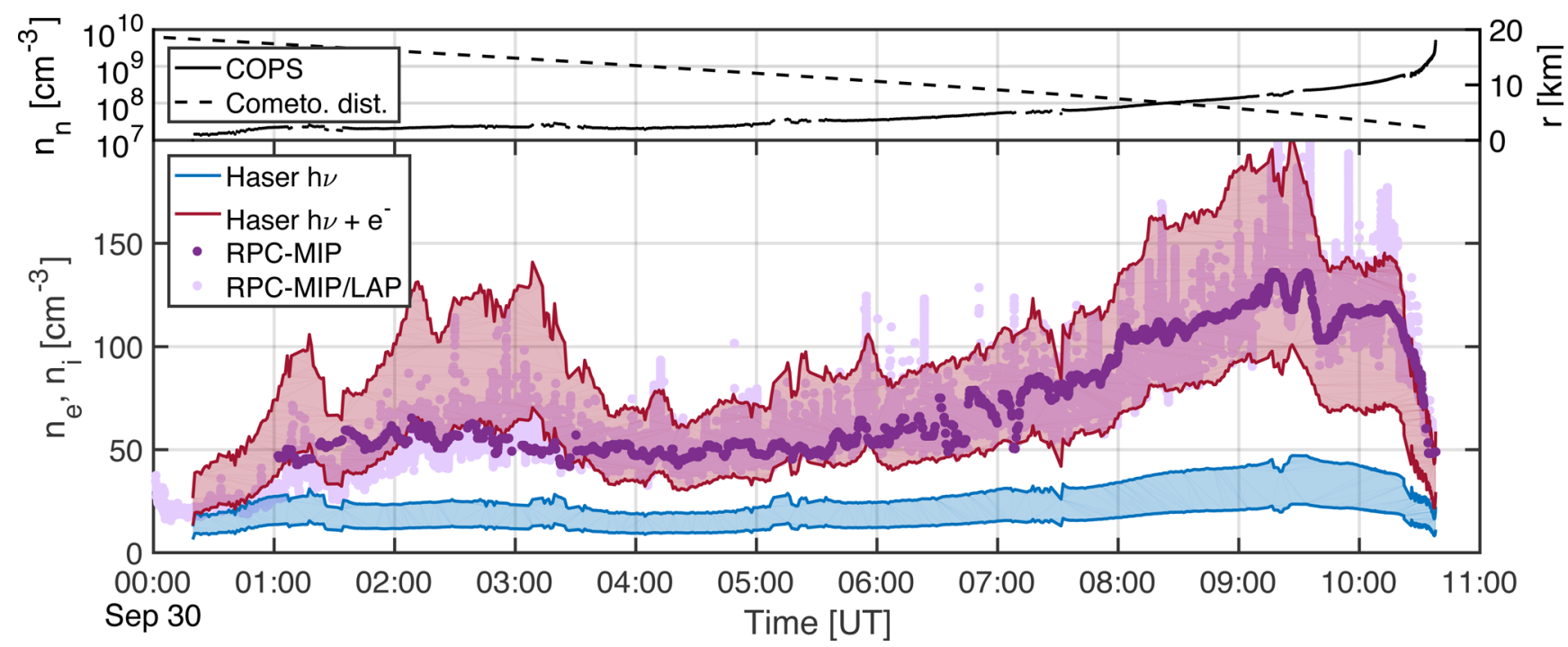

Figure 8. Top: Time series of the ROSINA-COPS measured neutral number density (full line) and cometocentric distance of the spacecraft (dashed line). Bottom: Time series of the RPC-MIP measured electron number density (purple dots), high time resolution RPC-LAP cross-calibrated with MIP (pink dots), details in Section 2. Simplified modelled ionospheric densities (equation 4) using photoionization only (blue) and both photoionization and electron-impact ionization (red), assuming outflow velocity from $400 \mathrm{~m} \mathrm{~s}^{-1}$ (upper bound) to $800 \mathrm{~m} \mathrm{~s}^{-1}$ (lower bound).

electron impact is also confirmed by the presence of some features in the RPC-MIP data set which are exclusively attributed to electronimpact ionization, such as the double hump in electron densities occurring at 09:30 UT. These humps cannot be associated with photoionization because the photoionization rate is constant throughout the day and there is no hump in the ROSINA-COPS neutral number density data set at that time. However, they are present on the time series of the electron-impact ionization frequencies in Fig. 6.

Around 03:00 UT, the modelled densities are overestimating the measured ionospheric densities. Looking at Fig. 6, it seems that there is an increase in the suprathermal electron flux. A possible explanation of the discrepancy could be that this electron flux is overestimated at lower cometocentric distance (it is assumed constant in the sub-spacecraft column, see Section 3.4.2). Note that this corresponds to times when the spacecraft was located above the neck of the nucleus, which was well illuminated, see Fig. 5. It is possible that an increased neutral activity over the neck induced some energy degradation of the suprathermal electron flux at closer cometocentric distances. Even though we are above the electron exobase, this would only concern electrons that have an energy well above average (above the $12 \mathrm{eV}$ ionization threshold of $\mathrm{H}_{2} \mathrm{O}$ ), to be differentiated from the bulk of the population $(\sim 2.75 \mathrm{eV})$ mentioned in Sections 2 and 3.5. The neck appears to be illuminated and visible again at 09:00 UT, but the same departure from the model cannot be seen due to the large uncertainty in $u$ (it is more visible with the refined model shown in Fig. 9). By computing the locally averaged plasma density, the role of this model is also to point out when plasma dynamics associated with local fluctuations take place. One can extract any departure from the local average to study these phenomena further by subtracting the modelled plasma densities to the measured electron number density from RPC-MIP and RPC-LAP. At any other time of the day, the model is able to explain the locally averaged ionospheric densities within the margin of error constrained by the neutral outflow velocities. Furthermore, the measured densities seem to be corresponding to the fast flow case during the first part of the descent $(r>10 \mathrm{~km})$ and slowly evolve to a slower flow representation as the spacecraft approaches the surface. The neutral flow is therefore accelerating as it is moving further away from the nucleus. As we are located below the ion exobase (see Section 3.5), the ions adopt the same velocity profile as the neutrals. The comparison between modelled densities and the data seem to confirm even more than this statement.

The margin of error given by the uncertainty in the outflow velocity is large. Besides, everything seems to point out that an acceleration of the neutrals and ions with increasing cometocentric distance takes place. In Section 4.2, we improve our model by modelling the outflow velocity as a function of the cometocentric distance.

\subsection{Ionopheric model with an $\boldsymbol{r}$-dependent velocity profile}

We refined our model by describing the neutral coma as an adiabatic expanding flow. We assume that the starting outflow velocity is $400 \mathrm{~m} \mathrm{~s}^{-1}$ with a temperature of $140 \mathrm{~K}$. Each neutral density profile is then calibrated with the real-time in situ ROSINA-COPS neutral density. It means that every ROSINA-COPS data point corresponds to a specific simulation for the neutrals. More details on this model are available in Heritier et al. (2017). Fig. 9 shows the ionospheric densities resulting from this approach with the black circles (including both photoionization and electron-impact ionization) compared with RPC-MIP measurements (purple dots) and high time resolution RPC-MIP/LAP measurements (pink dots). Apart from the brief overestimation at 03:00 and 09:00 uT, when Rosetta sees the illuminated neck (see Fig. 5), the agreement of the refined model with the RPC data set is excellent. The change in the neutral and ion outflow velocity is perfectly captured by the model. It is the constraint that was missing from the approach presented in Section 4.1.

From equation (3), one can deduce that the progressive increase of the measured and modelled ionospheric densities throughout the day is not only due to the local increase in neutral densities but also due to the fact that the local outflow velocity becomes slower at closer cometocentric distances. Indirectly, plasma density measurements, constrained with the neutral densities through a model, provide constraints on the neutral outflow velocities. Based on this quantitative comparison, the outflow velocity is estimated to be around 


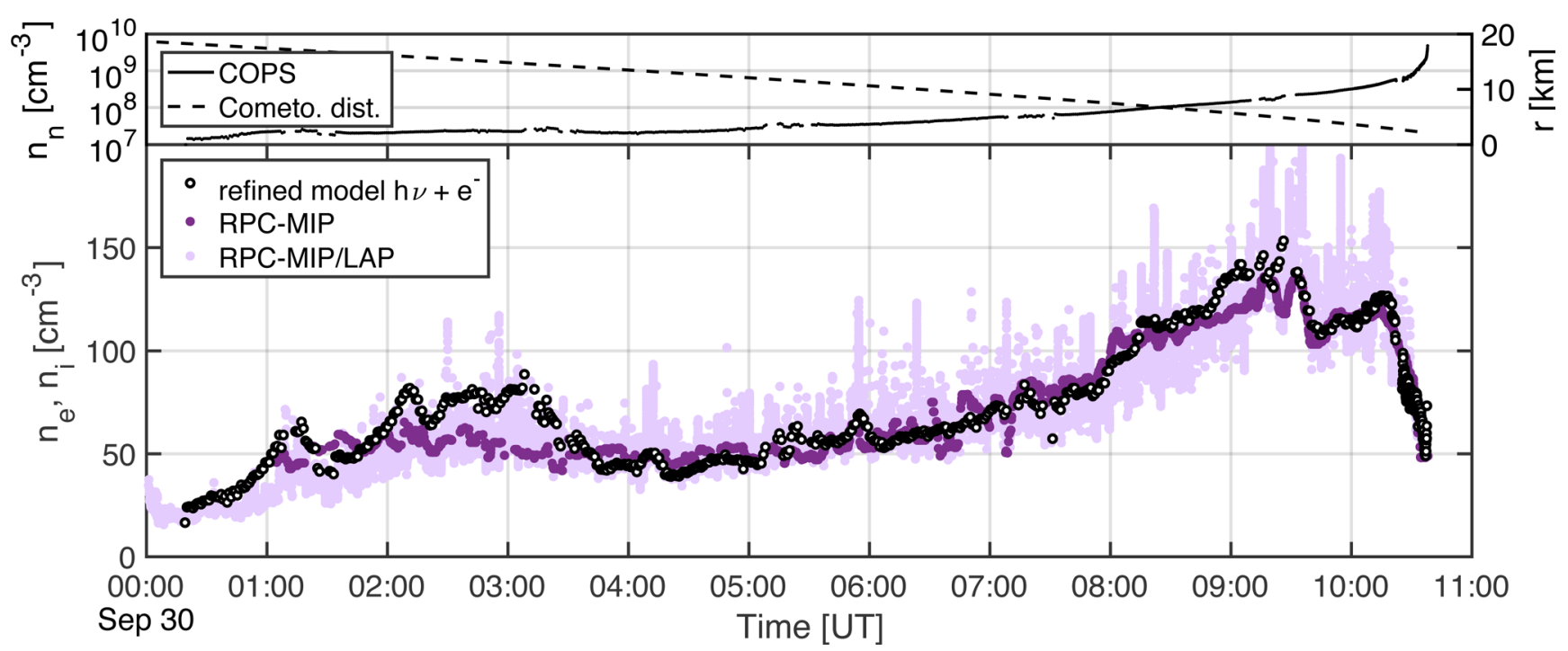

Figure 9. Top: Time series of the ROSINA-COPS measured neutral number density (full line) and cometocentric distance of the spacecraft (dashed line). Bottom: Time series of the RPC-MIP measured electron number density (purple dots, Section 2.1), high time resolution RPC-LAP cross-calibrated with MIP (pink dots, Section 2.3). Refined modelled ionospheric densities (equation 3) using a neutral gas expansion model calibrated on COPS measured densities (black circles).

$400 \mathrm{~m} \mathrm{~s}^{-1}$ near the surface of the nucleus and accelerates quickly to be about $800 \mathrm{~m} \mathrm{~s}^{-1}$ (terminal bulk velocity) at a cometocentric distance of $20 \mathrm{~km}$. This statement has to be moderated considering the fact that the latitude and longitude in the comet reference frame vary during the descent (see Figs 3 and 4), and the illumination and the sublimation process of the nucleus were therefore different. However, the constraints brought by a comparison between observed and modelled plasma densities are extremely valuable and show, in addition to the individual RPC-MIP and RPC-LAP data set, how powerful cross-calibration and multi-instrumental analysis can be.

At the very end of the descent, the ionospheric densities exhibit a local maximum (between 09:00 and 10:00 UT) of about $100 \mathrm{~cm}^{-3}$. This maximum was predicted by Vigren \& Galand (2013) and we can analytically estimate that it occurs at a cometocentric distance of twice the apparent radius of the nucleus (see Section 3.2). Following this peak, the ionospheric densities sharply decrease and it is perfectly captured by the model. During this short time span (after 10:00 UT), the neutral number density is however increasing drastically with decreasing altitude and the ion density source term of equation (1) is therefore increasing as well, proportionally with the neutrals. What is driving the ionopheric densities down is the fact that there is no ionization happening below the nucleus. At low cometocentric distances, the transport term bringing ions from even lower cometocentric distance becomes insignificant and would be zero in a control volume in contact with the surface. From a more algebraic point of view, the integral from $r_{0}$ to $r$ in equation (3) would be zero for $r=r_{0}$. This boundary condition is perfectly physical and is instrumentally observed for the first time in a cometary coma.

\section{CONCLUSION}

Rosetta's Grand Finale provided us with a unique opportunity to witness the expansion of a cometary ionosphere, offering us a unique and valuable data set: near-surface cometary plasma and its vertical structure. In this paper, we analysed the vertical structure - along the trajectory of Rosetta - of the ionospheric densities with crossinstrumental accuracy. We also identified the main drivers of the near-surface cometary ionosphere and quantified their contributions from our ionospheric model used as an organization element of a multi-instrumental data set.

RPC-MIP and RPC-LAP are complementary instruments. Combined together, RPC-MIP provided constraints on RPC-LAP which permitted to derive an estimate of the electron temperature (see Section 2.3). Over the final descent, we derived an average electron temperature of about $2.75 \pm 0.41 \mathrm{eV}$. Once RPC-LAP was calibrated, it provided us with a second estimation of the ionospheric densities. The RPC-MIP and cross-calibrated RPC-MIP/LAP electron density measurements are consistent with each other and provide a solid base for data comparison.

The key drivers to the ionospheric densities are outgassing rate, neutral outflow velocity, photoionization frequency and electronimpact ionization frequency. We assessed the ionospheric densities with three levels of accuracy:

(i) Assuming that these four drivers are constant over the different regions of the coma, the electron number density is predicted to be inversely proportional to the cometocentric distance (Edberg et al. 2015). When plotting the RPC-MIP and RPC-LAP electron densities as a function of the cometocentric distance (see Fig. 2), this trend is clearly noticeable but does not work very close from the nucleus. In fact, the real theoretical result should be proportional to $\left(r-r_{0}\right) / r^{2}$, where $r-r_{0}$ can be substituted by $r$ when $r>>r_{0}$ (Galand et al. 2016). But even if we correct for this factor, there will be errors induced by changes in illumination conditions and ionization frequencies. The most striking example occurs at 03:00 UT (or $15 \mathrm{~km}$ from the nucleus) where the $1 / r$ trend underestimates the electron densities (see Fig. 2). At the same time, the electron-impact ionization rates are larger than average (see Fig. 6). This feature, not captured by this simple model, explains the underestimation in terms of ion number densities.

(ii) We use instantaneous data inputs for the neutral number density (ROSINA-COPS) and the ionization frequency (daily TIMED/SEE for photoionization and instantaneous RPC-IES fluxes for electron-impact ionization) (Galand et al. 2016). This approach shown in Fig. 8 gives more accurate results and discard some of 
the discrepancies induced by spatial variations. Indeed, if a region has a higher outgassing rate for any reason (illumination, surface area seen from Rosetta, surface temperature, etc.), this will be taken into account in the neutral number density input of the model. This method also captures the peak in plasma density and some specific features, such as the double hump around 9:30 UT (Section 4.1). However, this model relies on a constant outflow velocity. From Fig. 8 showing the confrontation between the models and the RPC-MIP and LAP data, it seems that the outflow velocity is slower close to the nucleus than further away from the nucleus. Thus assuming a constant outflow velocity is not a suitable assumption in this configuration.

(iii) The refined approach applied for the first time to a multiinstrumental data set uses an adiabatic neutral profile to capture the acceleration of the neutral outflow as the cometocentric distance increases. The neutral outflow model is run for each ROSINACOPS data point to take into account the fact that the outflow velocity and the neutral profile might be different over the different regions covered by the spacecraft. We find excellent agreement with the RPC-MIP and RPC-LAP data set and very little margin of error compared with the previous approach. Some discrepancies remain persistent. The model is now slightly overestimating the neck region above which Rosetta passed at 03:00 UT. This could possibly be explained by variations in the electron energy flux along the subspacecraft column. We suspect the integrated flux to be lower at closer cometocentric distances than it is at the location of Rosetta.

Cometary vertical ionospheric models, as presented in Vigren \& Galand (2013), Vigren et al. (2015) and Galand et al. (2016), were verified for the first time near the nucleus surface. The maximum in ionospheric densities was predicted by Vigren \& Galand (2013) and is observed for the first time (see Figs 2, 8 or 9). Its theoretical value is found to be two times the apparent radius of the cometary nucleus (see Section 3.2). The sharp decrease following this maximum was also predicted by equation (4), first introduced by Galand et al. (2016). These ionospheric models are the proof that simple 1D models can successfully estimate ionospheric densities as long as they include the main physical drivers with good accuracy. They are also useful to identify secular or irregular events.

This study introduces the first ionospheric population data near the surface of a comet. Confronting them with the data acquired by the other RPC instruments will help us understand the remaining mysteries around cometary plasma. After Philae's descent in 2014 November and its onboard ROsetta MAgnetometer and Plasmamonitor (ROMAP), Auster et al. (2015) presented the first near-surface magnetic field data, proving that comets do not have an intrinsic magnetic field. The plasma monitor would be able to provide some information about the ionospheric population which would be very interesting to confront with the ones probed by the RPC-MIP and RPC-LAP instruments during the final descent of Rosetta.

\section{ACKNOWLEDGEMENTS}

Work at Imperial College London is supported by Imperial College President's scholarship, STFC of UK under grants ST/K001051/1 and ST/N000692/1 and ESA under contract No. 4000119035/16/ES/JD. Work at LPC2E/CNRS was supported by ESEP, CNES and ANR under the financial agreement ANR-15CE31-0009-01. The MIP-LAP cross-calibration was funded by ESA through the RPC Enhanced Archive activity. Work on ROSINA at the University of Bern was funded by the State of Bern, the Swiss National Science Foundation and the ESA PRODEX Program. The
Rosetta Plasma Consortium (RPC) instrument package consists of five sensors (RPC-ICA, RPC-IES, RPC-LAP, RPC-MAG, RPC$\mathrm{MIP}$ ) and a control unit (RPC-PIU). RPC is operated by Imperial College London in cooperation with SwRI, IRF, IGEP_TU Braunschweig, IRFU and LPC2E. The lead funding agencies are CNES, SNSB, NASA, BMWE and DLR, and STFC. We are indebted to the RPC and ROSINA operation and data processing teams. We are also very grateful to the TIMED/SEE PI, Tom Woods, and his team for providing us with the solar flux data set, associated routines of extrapolation to planets, and constructive insight on the solar data set [http://lasp.colorado.edu/see/]. We acknowledge the staff of CDDP and Imperial College London for the use of AMDA and the RPC Quicklook data base (provided by a collaboration between the Centre de Données de la Physique des Plasmas, supported by CNRS, CNES, Observatoire de Paris and Université Paul Sabatier, Toulouse and Imperial College London, supported by the UK Science and Technology Facilities Council). Data analysis was done with the QSAS science analysis system provided by the United Kingdom Cluster Science Centre (Imperial College London and Queen Mary, University of London) supported by The Science and Technology Facilities Council (STFC).

\section{REFERENCES}

Auster H.-U. et al., 2015, Science, 349

Balsiger H. et al., 2007, Space Sci. Rev., 128, 745

Bibring J.-P. et al., 2007, Space Sci. Rev., 128, 205

Bibring J.-P. et al., 2015, Science, 349, 493

Bieler A. et al., 2015, A\&A, 583, A7

Biver N. et al., 2015, A\&A, 583, A3

Broiles T. W. et al., 2016, MNRAS, 462, S312

Burch J. L., Goldstein R., Cravens T. E., Gibson W. C., Lundin R. N., Pollock C. J., Winningham J. D., Young D. T., 2007, Space Sci. Rev., 128, 697

Churyumov K. I., Gerasimenko S. I., 1972, in Chebotarev G. A., Kazimirchak-Polonskaia E. I., Marsden B. G., eds, Proc. IAU Symp. 45, The Motion, Evolution of Orbits, and Origin of Comets. Reidel Publishing Company, Dordrecht, p. 27

Clark G. et al., 2015, A\&A, 583, A24

Cravens T. E., 1997, Physics of Solar System Plasmas. Cambridge Univ. Press, Cambridge

Edberg N. J. T. et al., 2015, Geophys. Res. Lett., 42, 4263

Eriksson A. I. et al., 2007, Space Sci. Rev., 128, 729

Eriksson A. I. et al., 2017, A\&A

Fleshman B. L., Delamere P. A., Bagenal F., Cassidy T., 2012, J. Geophys. Res., 117, E05007

Fougere N. et al., 2016, MNRAS, 462, S156

Fuselier S. A. et al., 2015, A\&A, 583, A2

Fuselier S. A. et al., 2016, MNRAS, 462, S67

Galand M. et al., 2016, MNRAS, 462, S331

Gasc S. et al., 2017, Planet. Space Sci., 135, 64

Glassmeier K.-H., 2017, Phil. Trans. R. Soc. A, 375, 20160256

Glassmeier K.-H., Boehnhardt H., Koschny D., Kührt E., Richter I., 2007a, Space Sci. Rev., 128, 1

Glassmeier K.-H. et al., 2007b, Space Sci. Rev., 128, 649

Goldstein R. et al., 2015, Geophys. Res. Lett., 42, 3093

Gulkis S. et al., 2015, Science, 347, aaa0709

Hansen K. C. et al., 2016, MNRAS, 462, S491

Hässig M. et al., 2015, Science, 347, aaa0276

Henri P. et al., 2017, MNRAS, this issue

Heritier K. et al., 2017, MNRAS, in press

Itikawa Y., Mason N., 2005, J. Phys. Chem. Ref. Data, 34, 1

Lee S. et al., 2015, A\&A, 583, A5

Lemaire J., Scherer M., 1974, Space Sci. Rev., 15, 591

Madanian H. et al., 2016, J. Geophys. Res., 121, 5815

Mall U. et al., 2016, ApJ, 819, 126 
Mott-Smith H. M., Langmuir I., 1926, Phys. Rev., 28, 727

Nilsson H. et al., 2007, Space Sci. Rev., 128, 671

Nilsson H. et al., 2015a, Science, 347, aaa0571

Nilsson H. et al., 2015b, A\&A, 583, A20

Nilsson H. et al., 2017, MNRAS, this issue

Odelstad E. et al., 2015, Geophys. Res. Lett., 42, 10126

Odelstad E. et al., 2017, MNRAS, this issue

Rubin M., Hansen K. C., Gombosi T. I., Combi M. R., Altwegg K., Balsiger H., 2009, Icarus, 199, 505

Tawara H., 1978, At. Data Nucl. Data Tables, 22, 491

Trotignon J. G. et al., 2007, Space Sci. Rev., 128, 713

Vigren E., Galand M., 2013, ApJ, 772, 33

Vigren E., Galand M., Eriksson A. I., Edberg N. J. T., Odelstad E., Schwartz S. J., 2015, ApJ, 812, 54

Whipple F. L., Huebner W. F., 1976, ARA\&A, 14, 143

Woods T. N. et al., 2005, J. Geophys. Res., 110, A01312

Wurz P. et al., 2015, A\&A, 583, A22

\section{SUPPORTING INFORMATION}

Supplementary data are available at MNRAS online.

(i) illumination.mov displays the illuminated nucleus from the point of view of Rosetta during the final descent.

(ii) MIPdensity.avi displays the final trajectory of Rosetta with the associated plasma densities probed by RPC-MIP.

Please note: Oxford University Press is not responsible for the content or functionality of any supporting materials supplied by the authors. Any queries (other than missing material) should be directed to the corresponding author for the article.

This paper has been typeset from a $\mathrm{T}_{\mathrm{E}} \mathrm{X} / \mathrm{L} \mathrm{T} \mathrm{E} \mathrm{X}$ file prepared by the author. 